\title{
Revizyon diz artroplastisinde ekstansör mekanizma sorunları
}

\author{
Extensor mechanism problems in revision total knee arthroplasty
}

\author{
Cem Özcan, Mesut Tahta, Muhittin Şener
}

İzmir Katip Çelebi Üniversitesi, Atatürk Eğitim ve Araştırma Hastanesi, Ortopedi ve Travmatoloji Kliniği, İzmir, Türkiye

Revizyon diz artroplastisinin en ciddi komplikasyonlarından biri olan ekstansör mekanizma sorunları, klinik sonuçları doğrudan etkileyen önemli bir morbidite nedenidir. Karşılaşılabilecek önemli sorunlar; kuadriseps ve patellar tendon kopmaları, patella kırığı, patellar dizilim bozuklukları veya instabilitedir. Cerrah, hem yapılan ameliyatın morbiditesini azaltmak hem de olası birtakım hukuki problemlerden sakınmak için, olası komplikasyonları iyi bilmeli ve bunlarla başa çıkabilmelidir. Bu derlemede, revizyon diz artroplastisinde karşılaşılabilecek ekstansör mekanizma sorunlarının değerlendirilmesi ve çeşitli çözüm önerilerinin sunulması amaçlanmıştır.

Anahtar sözcülkler: revizyon, diz; artroplasti; ekstansör mekanizma; eklem instabilitesi
Extensor mechanism problems are one of the serious complications and an important cause of morbidity in revision knee arthroplasty, having direct consequences on clinical results. Serious complications such as quadriceps and patellar tendon rupture, patella fracture, patellar alignment problems and instability may be seen. In order to avoid legal problems and reduce morbidity rate, surgeons should know the possible complications of the operation and be able to manage them. In this review, causes and solutions of extensor mechanism problems are presented.

Key words: revision, knee; arthroplasty; extensor mechanism; joint instability
$\mathbf{R}$ evizyon diz artroplastisinin en ciddi komplikasyonlarından biri olan ekstansör mekanizma sorunları, klinik sonuçları doğrudan etkileyen önemli bir morbidite nedenidir. ${ }^{[1,2]}$

Kuadriseps tendonu veya patellar tendonun herhangi bir seviyesinden kopma olabilir. Önceki impantların çıkarılması sırasında zorlamaya ya da avasküler nekroza bağlı olarak gelişen patella kırığı, patellar dizilim bozuklukları ve instabilite, karşılaşılabilecek diğer önemli sorunlardır. ${ }^{[3-5]}$

Ortopedik cerrah, hem yapılan ameliyatın morbiditesini azaltmak hem de olası birtakım hukuki problemlerden sakınmak amacıyla, olası komplikasyonları iyi bilmeli ve bunlarla başa çıkabilmelidir.

Bu derlemede revizyon diz artroplastisinde karşılaşılabilecek ekstansör mekanizma sorunlarının değerlendirilmesi ve çeşitli çözüm önerilerinin sunulması amaçlanmıştır.

\section{EKSTANSÖR MEKANIZMA DEVAMLILIĞININ BOZULMASI}

Ekstansör mekanizma bütünlügünün bozulması, çeşitli nedenlerle olabilir. Ciddi sonuçlarla karşılaşılabilmesi nedeniyle birincil amaç, bu komplikasyona yol açmamak olmalıdır. ${ }^{[6,7]}$ Ekstansör mekanizma bütünlüğü, anatomik dizilimi boyunca herhangi bir yerden bozulabilir.

\section{Tendon Rüptürleri}

\section{Patellar tendon rüptürü}

Sert dizlerde, özellikle de patella baja (Şekil 1) varlı̆̆ında, dikkat edilmediği takdirde patellar tendon rüptürü gelişebilir. Ayrıca, travmatik hiperfleksiyon zorlaması, ameliyat sırasında uygulanan sert manipülasyonlar, steroid kullanımı ve önceki cerrahi girişimler de patellar tendon rüptürlerinin etiyolojisinde sıklıkla karşımıza çıkmaktadır. ${ }^{[8,9]}$ Patellar tendon, en sık tibiaya yapışma

- İletişim adresi: Dr. Muhittin Şener, İzmir Katip Çelebi Üniversitesi, Atatürk Eğitim ve Araştırma Hastanesi, Ortopedi ve Travmatoloji Kliniği, İzmir, Türkiye Tel: 0532 - 3523628 e-posta: msener87@yahoo.com

- Geliștarihi: 8 Nisan $2015 \quad$ Kabul tarihi: 8 Nisan 2015 


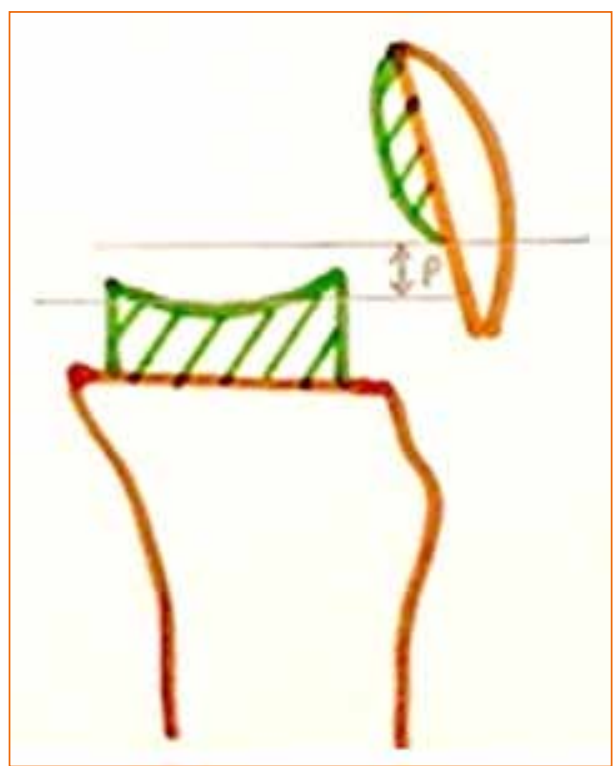

Şekil 1. Patella baja varlığında patellar sıkışma.

yerinden ayrilsa da, herhangi bir seviyeden kopabilir. Dizin bu bölgesinin yumuşak doku desteği oldukça zayıftır ve cerrah, mutlaka ilave yumuşak doku örtümü seçeneklerini de göz önünde bulundurmalıdır. ${ }^{[10,11]}$

Patellar tendon rüptürlerinde pek çok tedavi seçeneği mevcut olsa da, her şeyden önce komplikasyon oranının yüksek olduğu ve ideal ekstansör mekanizmanın nadiren restore edilebildiği unutulmamalıdır. Kemikten olan akut avulsiyonlar, tibiaya dril ucu ile açılan deliklerden geçen sütürler ya da sütür ankorlar ile primer onarılabilir. Tendon üzerinde rüptür varsa, yine primer onarım denenebilir. Buna rağmen, tibial tüberkülden olan avulsiyonların sonuçları oldukça kötüdür. Rang ve arkadaşları, hangi tespit materyali kullanılırsa kullanısın, \%90 başarısızlık bildirmişlerdir. ${ }^{[12]}$ Her ne kadar Mittal ve arkadaşları ${ }^{[13]}$ augmentasyon yapılmadan onarım tarif etmişlerse de, semitendinöz veya gracilis tendonları ile augmentasyon mutlaka düşünülmelidir. Bunu yaparken, Cadambi ve arkadaşlarının tanımladığı gibi, kullanılan tendonun distal yapışma yeri intakt bırakılarak, proksimalde kastan ayrıldıktan sonra patellaya açılan delikten geçirilmeli ve patellar tendon distal yumuşak doku kitlesine dikilmelidir. ${ }^{[14]}$ Kronik patellar tendon rüptürlerinde ise durum daha karmaşıktır. Tendondaki kısalma nedeniyle, uygun onarımın sağlanması oldukça zordur. Bu nedenle, patella seviyesinde sorun yoksa, sık tercih edilen yöntemlerden biri, kemik blok içeren aşil tendon allogrefti kullanımıdır. ${ }^{[15]}$ Malhotra ve arkadaşlarının önerdiği şekilde, ön çapraz bağ rekonstrüksiyonlarında kullanılan bone-patellar tendon-bone (BTB) allogrefti de kullanılabilir. ${ }^{16]}$ Whiteside ve arkadaşları ise, ekstansör mekanizmanın defektli olduğu durumlarda vastus mediyalis ve lateralis flebinin kullanımını tanımlamışlardır. ${ }^{[17]}$ Uzun dönem sonuçları henüz bilinmemekle birlikte, Browne ve arkadaşları, daha yeni bir yöntem olan monofilaman poliprolen sentetik mesh kullanımını tanımlamışlardır.[18] Tendon rüptürüne patellar parçalanmanın eşlik etmesi ve onarılamayacak kadar kötü kemik varlığı ya da herhangi bir nedenle tüm bu yöntemlerin uygulanamadığı durumlarda, Helito ve arkadaşları ile Brown ve arkadaşlarının önerdiği şekilde, ekstansör mekanizma allogrefti kullanılabilir. ${ }^{[19-21]}$

Bahsedilen tedavi seçenekleri, patellar tendon rüptürlerinde tam restorasyonu genellikle sağlayamamaktadır. Bu noktada en iyi tedavinin, bu durumun önlenmesi olduğu unutulmamalıdır. ${ }^{[22]} \mathrm{Bu}$ konuda yapılabileceklerin başında, revizyon cerrahisi sırasında ekstansör mekanizmaya hasar vermeden dizin ortaya konulması gelmektedir. Bunun için, eski insizyonun 4-5 cm uzatılması, yeterli kapsüler gevşetme yapılması ve gerektiği durumlarda patellar tendonun tibial tüberküle yapışma noktasına destek pini konması gibi yöntemlerin yanında, Lahav ve arkadaşlarının ${ }^{[23]}$ tanımladığı gibi geniş gevşetmelerin kullanıldığı yöntemler tercih edilebilir. ${ }^{[24]}$ Tüm bunlara rağmen, özellikle sıkı dizlerde, revizyon cerrahisi sırasında patellanın devrilmesi mümkün olmayabilir. Böyle durumlarda, literatürde proksimal ve distal gevşetmeler tanımlanmıştır. Proksimal gevşetmelerden biri olan, rectus femoris'in muskülotendinöz bileşkesinden $\mathrm{V}$ insizyonla ayrılıp patellanın devrilmesini sağlayan "turndown" metodu (Şekil 2), mediyal ve lateral superior genikulat arterleri korumadığından dolayı, nadiren kullanılmaktadır. ${ }^{[25]}$ Bu yöntem, lateral superior arteri korumak amacıyla modifiye edilmiş, ${ }^{[26]} \mathrm{V}$ insizyonun laterale uzanan kolu bir miktar yumuşak doku bırakılarak sonlandırılmıştır (Şekil 3). Bir diğer yöntem olan "rectus snip"te, rectus femoris muskülotendinöz bileşkesine kısa proksimal lateral oblik insizyon uygulanarak, patellanın devrilmesi sağlanır (Şekil 4). ${ }^{[27]}$ Distal gevşetmelerden olan proksimal tibial tüberkül osteotomisinin, çeşitli modifikasyonları vardır. Bunlardan en popüler olanı, tibial krestin mediyalden kesilip laterale ilerletildiği yöntemdir (Şekil 5). ${ }^{[28]}$ Ayrılan kemik parçanın tespiti vida veya tel ile yapılabilmekle birlikte, tel ile tespit sonrasında erken harekete başlanabileceğini ve daha avantajlı olduğunu bildiren yayınlar mevcuttur. ${ }^{[29]}$

\section{Kuadriseps tendon rüptürü}

Kuadriseps tendon rüptürü, genellikle travma sonrası oluşur. Sistemik hastalıklar, önceki cerrahi girişimlerde kuadriseps snip veya turndown uygulanmış olması ya da eklem hattı seviyesinin değişmiş olması gibi predispozan etmenlerin varlığında, rüptür riski artar. [6,30] Tedavi yöntemine karar vermede; yaralanmanın 


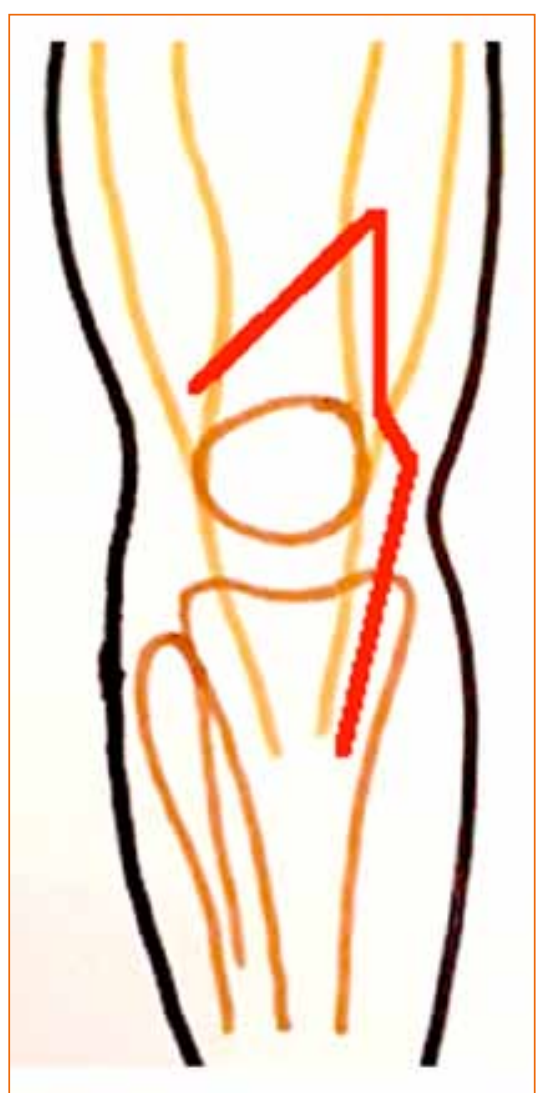

Şekil 2. Turndown metodu.

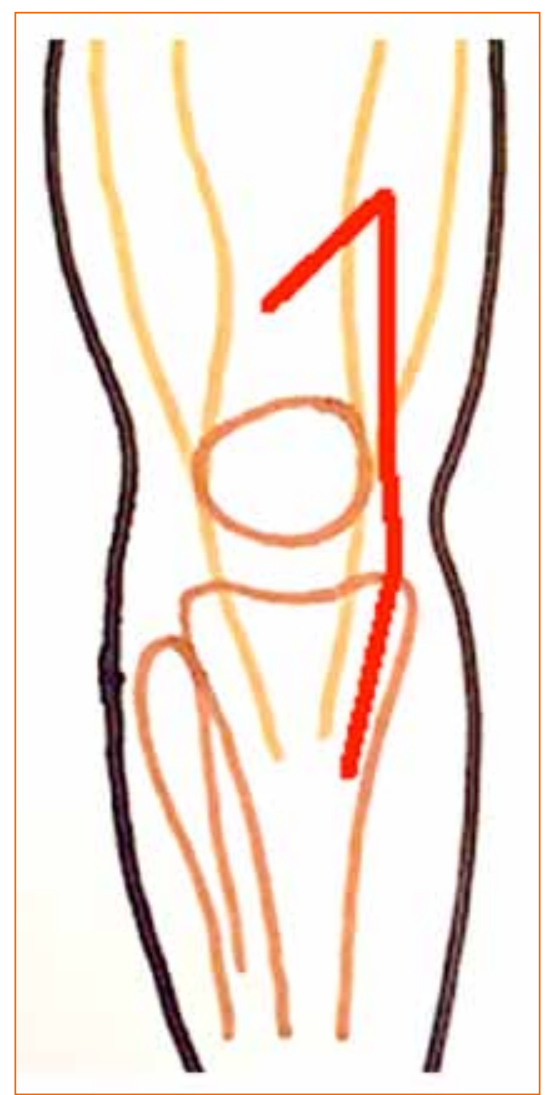

Şekil 3. Modifiye turndown metodu.

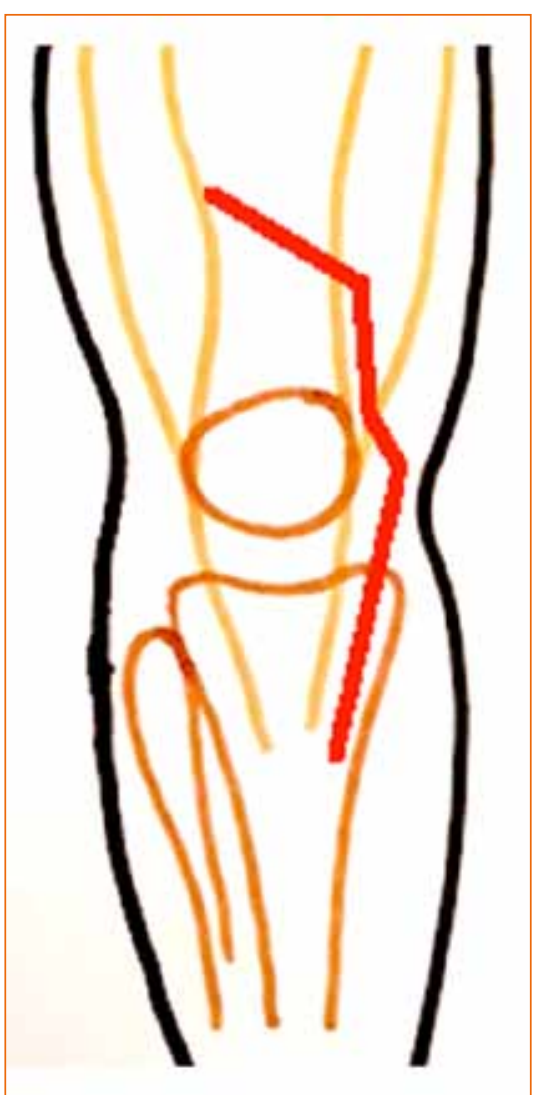

Şekil 4. Rectus snip metodu.

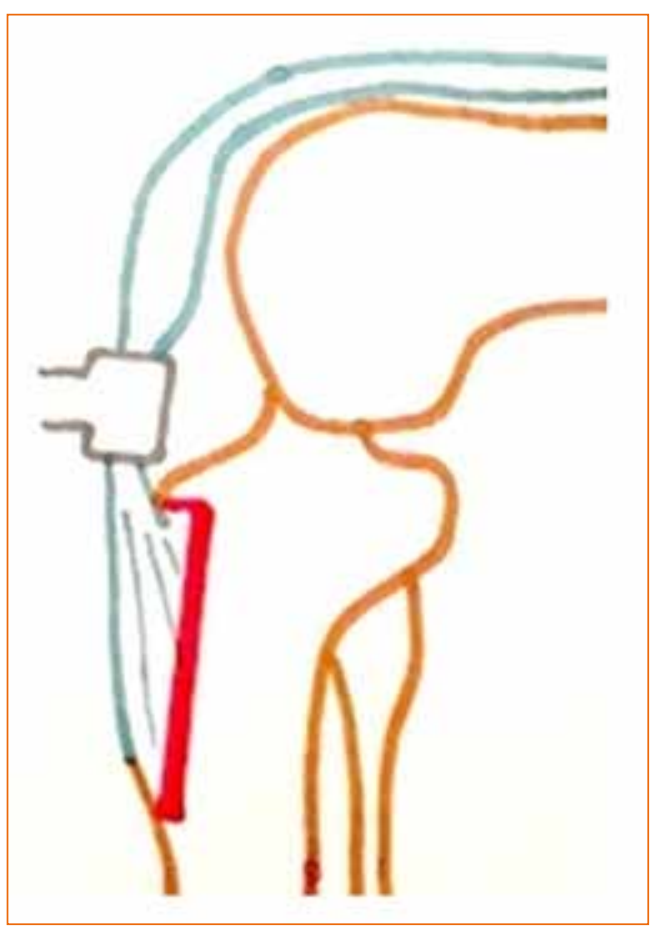

Şekil 5. Tibial tüberkül osteotomisi. niteliği, kalan tendonun kalitesi ve kemik yapıların bütünlüğü gibi durumlar göz önünde bulundurulmalıdır.

Kısmi akut kuadriseps tendon rüptürlerinde, Dobbs ve arkadaşları, immobilizasyon sonrası breys kontrolünde kademeli aktif fleksiyon ile yapılan konservatif tedavinin genellikle etkin olduğunu bildirmişlerdir. ${ }^{[6]}$ Total akut kuadriseps tendon rüptürlerinde ise, onarım veya çeşitli greft seçenekleri kullanılarak cerrahi tedavi tercih edilmelidir. Tedavide, patellar tendon avulsiyonlarında olduğu gibi, patellaya matkap ucu ile açılan delikler kullanılarak veya sütür ankorlar ile primer onarım uygulanabilir; ${ }^{[31]}$ ancak, Lynch ve arkadaşları, kalıcı ekstansör kayıp gelişebileceğini bildirmişlerdir. ${ }^{[32]}$ Kronik olgularda ise, rerüptür riski \%33-36 civarındadır. ${ }^{[33]}$ Tedavide geç kalındığında kuadriseps kasının retrakte olacağı unutulmamalı, mümkün olan en kısa sürede rekonstrüksiyon yapılmalıdır. Bu durumda seçilecek yöntemler; kuadriseps V-Y plasti, allogreft ile rekonstrüksiyon, semitendinozus veya grasilis ile augmentasyon ya da sentetik greft ile rekonstrüksiyon olmalıdır. ${ }^{[3,4,34]}$ Bununla birlikte, hangi greft yönteminin daha iyi sonuç vereceğini, günümüz literatürüne göre belirlemek henüz mümkün değildir. 


\section{Patella Kırığı}

Patella kırı̆ı, genellikle travma sonucu veya kuadrisepsin ani eksentrik kasılması ile oluşmaktadır. Etiyolojisinde pek çok predispozan etmen vardır. Genellikle, 1) hastaya (obezite, yüksek aktivite düzeyi, ileri fleksiyon dereceleri, osteopeni), 2) implanta (patellar komponent varlığı, merkezi tek pegli patellar implant kullanımı, patellar komponentin metal yüzeyli - çimentosuz olması) ve 3) tekniğe bağlı (patellar dizilim bozukluğu, uygunsuz kalınlıkta implant kullanımı, ısı nekrozu, aşırı kuadriseps gevşetme) olmak üzere, üç kategoride değerlendirilmektedir. ${ }^{[35]}$

Patella kırıklarının yönetiminde, patellar komponentte gevşeme olup olmadığı oldukça önemlidir. Ortiguera ve arkadaşları, komponent gevşemesi olmayan ve ekstansör mekanizmanın intakt olduğu durumlarda, dizi immobilize edip kademeli hareket vererek oldukça iyi sonuçlar elde etmişlerdir. ${ }^{[36]}$ Yirmi derecenin üzerinde ekstansör kayıp olan olgularda ise, cerrahi tedavi tercih edilmelidir. ${ }^{[3]}$ Kırık mevcutsa; küçük parçalar eksize edilip, büyükler fragmanlar fikse edilmelidir; yumuşak doku onarımı yapılmalı ve gevşek patellar implant varlığında yeterli kemik stoğu varsa, patellar implant revize edilmelidir. ${ }^{[4]}$ Gevşemiş komponent varlığında, öncelikle bunun nedeni araştırılmalı, patella revizyonundan önce bu sorun çözülmelidir. ${ }^{[37]}$ Patellar implant gevşekse çıkarılmalı ve kalan kemik stoğu değerlendirilmelidir. Bazen ince, yetersiz ve kötü kalitede bir kemikle karşılaşılabilir. Kemik parçalarda avasküler nekroz olabileceği ve güvenli internal tespitin zor olabileceği mutlaka göz önünde bulundurulmalıdır. Böyle durumlarda, Reuben ve arkadaşları, genellikle transvers patella kemik stoğu 15 $\mathrm{mm}$ 'den daha fazla ise patellar komponentin revizyonunu, daha az ise artmış kırık riski nedeniyle implant yerleştirmeden çok patelloplastinin tercih edilmesi gerektiğini bildirmişlerdir. ${ }^{[38]}$ Rekonstrüksiyon sağlanamazsa, bir diğer seçenek olarak patellektomi kullanılabilir. ${ }^{29,39]}$

\section{DIZILIM BOZUKLUKLARI VE PATELLAR INSTABILITE}

Eklem hattının uygun restorasyonu, patellofemoral uyum ve dengenin kurulabilmesinin vazgeçilmez şartlarından biridir. Eklem hattının yükselmesi, patella bajaya neden olur ve özellikle fleksiyonda tibial insert ile sürtünme nedeniyle, ön diz ağrısı ile fleksiyonda azalmaya neden olur. Bu değişiklik $8 \mathrm{~mm}$ 'den fazla olursa, klinik sonuçlara da yansır. ${ }^{[40-42]}$ Işık ve arkadaşları, eklem hattının distale inmesi sonucunda, özellikle lateralde hissedilen diz ağrısı oluşabileceğini bildirmişlerdir. ${ }^{[43]}$ ideal eklem hattının belirlenmesinde kullanılacak teknikle ilgili olarak literatürde uzlaşı olmasa da adduktor tüberkül, mediyal epikondil, lateral epikondil veya fibula başı kullanılabilir. ${ }^{[4,45]}$ Revizyon diz artroplastisinde, kemik defektleri, osteoliz ve önceki implantların çıkarılması sırasında oluşan defektler nedeniyle, bu anatomik lokalizasyonlardan uygun olan herhangi biri kullanılabilir. Bu da mümkün değilse, hastanın implant yerleştirilmeden önceki grafileri veya karşı dizin grafileri yararlı olabilir.

Distal femurdaki osteoliz nedeniyle veya implantlar çıkarılırken kemiğe verilen hasar sonucu kolaylıkla patella baja ile karşılaşılabilinir. Böyle durumlarda belirlenen eklem seviyesine ulaşmak için bloklar veya kamalar kullanılabilir. Aynı nedenlerle tibial defektlerin eklem hattını distale ilerletmesini engellemek için bloklar, kamalar kullanılabilir ya da insert kalınlığı arttırılabilir. Daha kompleks durumlarda patellar implantın yerini uygun yere ilerletmek ya da tibial tüberkül osteotomisi ile tüberkülün yerini değiştirmek yarar sağlayabilir. ${ }^{[29]}$

Kalın patellar komponent seçilmesi ya da komponentin lateralize yerleştirilmesi, femoral ve tibial komponentlerin iç rotasyonu, tibiofemoral dizilimin valgusta olması, femoral komponentin mediyalizasyonu gibi cerrahi teknikteki hatalar, patellar subluksasyon veya dislokasyonla sonuçlanabilir. Lateral dokulardaki gerginlik de bir başka önemli predispozan etmendir. Bu nedenle, cerrahi sırasında denemelerle değerlendirme oldukça önemlidir ve bahsedilen nedenler gözden geçirilerek anında çözüm geliştirilebilir. Cerrahi sonrası dönemde farkına varıldığında ise, fizik tedavi veya breys kullanımı gibi konservatif tedavilerin başarısı ne yazık ki oldukça düşüktür. Bu durumda, çözüm yine etiyolojiye yönelik olmalıdır. ${ }^{[9,32,46,47]}$ Komplikasyon komponent malpozisyonuna bağlı ise, uygun dizilim ve rotasyon yeniden sağlanmalıdır; lateral dokulardaki gerginliğe bağlı ise, artroskopik veya açık lateral retinakuler gevşetme yapılabilir. ${ }^{[29]}$

\section{SONUÇ}

Revizyon diz artoplastisinde ekstansör mekanizma sorunları, cerrahi sonrası ciddi komplikasyonları nedeniyle, mümkün olduğunca erken ve uygun tedavi edilmelidir. Her ne kadar genellikle cerrahi tedavi gerektirse de, konservatif tedavinin güvenle kullanılabileceği durumlar vardır. Günümüzde kabul edilen en yaygın görüşe göre; en iyi tedavi, ekstansör mekanizma komplikasyonlarının oluşmadan önlenmesidir; tedaviyi yönlendirirken, sorunun lokalizasyonu, implantların gevşeyip gevşemediğinin, yumuşak dokuların kalitesinin ve hastanın genel durumunun bir arada düşünülmesi gerektiği akıldan çıkarılmamalıdır. 


\section{KAYNAKLAR}

1. Doolittle $\mathrm{KH}$ 2nd, Turner RH. Patellofemoral problems following total knee arthroplasty. Orthop Rev 1988;17(7):696-702.

2. MacCollum MS 3rd, Karpman RR. Complications of the PCA anatomic patella. Orthopedics 1989;12(11):1423-8.

3. Park SS, Kubiak EN, Wasserman B, Sathappan SS, Di Cesare PE. Management of extensor mechanism disruptions occurring after total knee arthroplasty. Am J Orthop (Belle Mead NJ) 2005;34(8):365-72.

4. Cooney WP 4th, Sierra RJ, Trousdale RT, Pagnano MW. Revision total knees done for extensor problems frequently require reoperation. Clin Orthop Relat Res 2005;440:117-21.

5. Goldberg VM, Figgie HE 3rd, Inglis AE, Figgie MP, Sobel M, Kelly M, Kraay M. Patellar fracture type and prognosis in condylar total knee arthroplasty. Clin Orthop Relat Res 1988;(236):115-22.

6. Dobbs RE, Hanssen AD, Lewallen DG, Pagnano MW. Quadriceps tendon rupture after total knee arthroplasty. Prevalence, complications, and outcomes. J Bone Joint Surg Am 2005;87(1):37-45.

7. Healy WL, Wasilewski SA, Takei R, Oberlander M. Patellofemoral complications following total knee arthroplasty. Correlation with implant design and patient risk factors. J Arthroplasty 1995;10(2):197-201.

8. Kelly MA. Patellofemoral complications following total knee arthroplasty. Instr Course Lect 2001;50:403-7.

9. Parker DA, Dunbar MJ, Rorabeck $\mathrm{CH}$. Extensor mechanism failure associated with total knee arthroplasty: prevention and management. J Am Acad Orthop Surg 2003;11(4):238-47.

10. Rosenberg AG. Management of extensor mechanism rupture after TKA. J Bone Joint Surg Br 2012;94(11 Suppl A):116-19. CrossRef

11. Busfield BT, Huffman GR, Nahai F, Hoffman W, Ries MD. Extended medial gastrocnemius rotational flap for treatment of chronic knee extensor mechanism deficiency in patients with and without total knee arthroplasty. Clin Orthop Relat Res 2004;(428):190-7.

12. Rand JA, Morrey BF, Bryan RS. Patellar tendon rupture after total knee arthroplasty. Clin Orthop Relat Res 1989;(244):233-8.

13. Mittal R, Kumar N, Yadav C, Kumar A. Direct Repair without Augmentation of Patellar Tendon Avulsion following TKA. Case Rep Orthop 2015;2015:391295. CrossRef

14. Cadambi A, Engh GA. Use of a semitendinosus tendon autogenous graft for rupture of the patellar ligament after total knee arthroplasty. J Bone Joint Surg Am 1992;74(7):974-9.

15. Crossett LS, Sinha RK, Sechriest VF, Rubash HE. Reconstruction of a ruptured patellar tendon with achilles tendon allograft following total knee arthroplasty. J Bone Joint Surg Am 2002;84-A(8):1354-61.

16. Malhotra R, Garg B, Logani V, Bhan S. Management of extensor mechanism deficit as a consequence of patellar tendon loss in total knee arthroplasty: a new surgical technique. J Arthroplasty 2008;23(8):1146-51. CrossRef

17. Whiteside LA. Surgical technique: vastus medialis and vastus lateralis as flap transfer for knee extensor mechanism deficiency. Clin Orthop Relat Res 2013;471(1):221-30. CrossRef

18. Browne JA, Hanssen AD. Reconstruction of patellar tendon disruption after total knee arthroplasty: results of a new technique utilizing synthetic mesh. J Bone Joint Surg Am 2011;93(12):1137-43. CrossRef
19. Helito CP, Gobbi RG, Tozil MR, Félix M, Angelini FJ, Pécora RJ. Extensor mechanism allograft in total knee arthroplasty. Acta Ortop Bras 2013;21(6):315-9. CrossRef

20. Brown NM, Murray T, Sporer SM, Wetters N, Berger RA, Della Valle CJ. Extensor mechanism allograft reconstruction for extensor mechanism failure following total knee arthroplasty. J Bone Joint Surg Am 2015;97(4):279-83. CrossRef

21. Emerson RH Jr, Head WC, Malini TI. Reconstruction of the patellar tendon rupture after total knee arthroplasty with an extensor mechanism allograft. Clin Orthop Relat Res 1990;(260):154-61.

22. Parker DA, Dunbar MJ, Rorabeck CH. Extensor mechanism failure associated with total knee arthroplasty: prevention and management. J Am Acad Orthop Surg 2003;11(4):238-47.

23. Lahav A, Hofmann AA. The "banana peel" exposure method in revision total knee arthroplasty. Am J Orthop (Belle Mead NJ) 2007;36(10):526-9.

24. Nam D, Abdel MP, Cross MB, LaMont LE, Reinhardt KR, McArthur BA, Mayman DJ, Hanssen AD, Sculco TP. The management of extensor mechanism complications in total knee arthroplasty. AAOS Exhibit selection. J Bone Joint Surg Am 2014;96(6):e47. CrossRef

25. Coonse J, Adams JD. A new operative approach to the knee joint. Surg Gynecol Obstet 1943;77:334-40.

26. Trousdale RT, Hanssen AD, Rand J, Calahan TD. V-Y quadricepsplasty in total knee arthroplasty. Clin Orthop Relat Res 1993;(286):48-55.

27. Garvin KL, Scuderi G, Insall JN. Evolution of the quadriceps snip. Clin Orthop Relat Res 1995;(321):131-7.

28. Barrack RL, Smith P, Munn B, Engh G, Rorabeck C. The Ranawat Award. Comparison of surgical approaches in total knee arthroplasty. Clin Orthop Relat Res 1998;(356):16-21.

29. Laskin RS, Beksaç B. Management of the Extensor Mechanism During Revision Total Knee Arthroplasty. In: Bono JV, Scott RD, editors. Revision Total Knee Arthroplasty. New York: Springer; 2005. p.152-67.

30. Shah MK. Simultaneous bilateral rupture of quadriceps tendons: analysis of risk factors and associations. South Med J 2002;95(8):860-6.

31. Kim TW, Kamath AF, Israelite CL. Suture anchor repair of quadriceps tendon rupture after total knee arthroplasty. J Arthroplasty 2011;26(5):817-20. CrossRef

32. Lynch AF, Rorabeck CH, Bourne RB. Extensor mechanism complications following total knee arthroplasty. J Arthroplasty 1987;2(2):135-40.

33. Yun AG, Rubash HE, Scott RD, Laskin RS. Quadriceps rupture associated with a proximal quadriceps release in total knee arthroplasty. A report of three cases. J Bone Joint Surg Am 2003;85-A(9):1809-11.

34. Pagnano MW. Patellar tendon and quadriceps tendon tears after total knee arthroplasty. J Knee Surg 2003;16(4):242-7.

35. Parvizi J, Kim KI, Oliashirazi A, Ong A, Sharkey PF. Periprosthetic patellar fractures. Clin Orthop Relat Res 2006;446:161-6.

36. Ortiguera CJ, Berry DJ. Patellar fracture after total knee arthroplasty. J Bone Joint Surg Am 2002;84-A(4):532-40.

37. Rosenberg AG, Jacobs JJ, Saleh KJ, Kassim RA, Christie MJ, Lewallen DG, Rand JA, Rubash HE. The patella in revision total knee arthroplasty. J Bone Joint Surg Am 2003;85-A Suppl 1:S63-70.

38. Reuben JD, McDonald CL, Woodard PL, Hennington LJ. Effect of patellar thickness on patellar strain after total knee arthroplasty. J Arthroplasty 1991;6(3):251-8. 
39. Chang MA, Rand JA, Trousdale RT. Patellectomy after total knee arthroplasty. Clin Orthop Relat Res 2005;440:175-7.

40. Paulos LE, Pinkowski JL. Patella infera. In: Fox JM, Del Pizzo W, editors.The Patellofemoral Joint. New York: McGraw-Hill Inc.; 1993. p.205-14.

41. Engh GA, McAuley JP. Joint line restoration and flexionextension balance during revision total knee arthroplasty. In: Engh GA, Rorabeck $\mathrm{CH}$, editors. Revision Total Knee Arthroplasty: Techniques in Revision Surgery. Baltimore: Williams and Wilkins; 1997. p.234-51.

42. Partington PF, Sawhney J, Rorabeck CH, Barrack RL, Moore J. Joint line restoration after revision total knee arthroplasty. Clin Orthop Relat Res 1999;(367):165-71.

43. Isik C, Dasar U, Tahta M, Cay N, Akmese R, Isik D, Bozkurt $M$. The relationship between incorrect restoration of the joint line and lateral knee pain in patients undergoing total knee arthroplasty. Open J Rheumatol Autoimmune Dis 2014;4:138-45. CrossRef
44. Romero J, Seifert B, Reinhardt O, Ziegler O, Kessler O. A useful radiologic method for preoperative joint-line determination in revision total knee arthroplasty. Clin Orthop Relat Res 2010;468(5):1279-83. CrossRef

45. Martin JW, Whiteside LA. The Influence of joint line position on knee stability after condylar knee arthroplasty. Clin Orthop Relat Res 1990;(259):146-56.

46. Anderson JA, Baldini A, Sculco TP. Patellofemoral function after total knee arthroplasty: a comparison of 2 posteriorstabilized designs. J Knee Surg 2008;21(2):91-6.

47. Akagi $M$, Matsusue $Y$, Mata $T$, Asada $Y$, Horiguchi $M$, lida $\mathrm{H}$, Nakamura T. Effect of rotational alignment on patellar tracking in total knee arthroplasty. Clin Orthop Relat Res 1999;(366):155-63. 